\title{
Effects of Ibudilast on the Subjective, Reinforcing, and Analgesic Effects of Oxycodone in Recently Detoxified Adults with Opioid Dependence
}

\author{
Verena E Metz",', Jermaine D Jones', Jeanne Manubay', Maria A Sullivan',2, Shanthi Mogali', Andrew Segoshi', \\ Gabriela Madera', Kirk W Johnson ${ }^{3}$ and Sandra D Comer' \\ 'Department of Psychiatry, Columbia University Medical Center, New York State Psychiatric Institute, New York, NY, USA; ${ }^{2}$ Clinical Research and \\ Development, Alkermes, Waltham, MA, USA; ${ }^{2}$ Development, XOMA Corporation, Berkeley, CA, USA
}

\begin{abstract}
Ibudilast, a nonselective phosphodiesterase inhibitor, is used clinically in Asia for the treatment of asthma and poststroke dizziness. Recent preclinical studies have suggested that it also inhibits glial cell activation in rodents, and may alter opioid-mediated effects, including analgesia and withdrawal symptoms. The effects of ibudilast on the abuse potential of opioids in humans are largely unknown. The present study was designed to examine the influence of ibudilast on subjective (including drug craving), reinforcing, and analgesic effects of oxycodone in human volunteers diagnosed with opioid dependence (equivalent to moderate-severe opioid use disorder). Non-treatment-seeking opioiddependent male volunteers $(n=1 \mathrm{I})$ underwent an in-patient detoxification with morphine, followed by maintenance on placebo ( 0 mg b.i.d.) and active ibudilast (50 mg b.i.d.). Under each maintenance dose, six experimental sample and choice sessions were completed involving oral oxycodone administration (0, 15, and $30 \mathrm{mg} / 70 \mathrm{~kg}$, p.o.). Subjective effects of oxycodone and drug craving were measured with visual analog scales (VAS) and a Drug Effects Questionnaire. The cold pressor test was used to produce pain, and a modified progressive-ratio choice procedure was used to measure the reinforcing effects of oxycodone. Under the active ibudilast condition compared with the placebo condition, ratings of drug liking following $15 \mathrm{mg}$ of oxycodone were decreased significantly. The mean drug breakpoint value was also significantly lower in the active vs the placebo ibudilast condition under the $15 \mathrm{mg}$ oxycodone condition, but not significantly lower under the $30 \mathrm{mg}$ oxycodone condition. Heroin craving was significantly reduced under active ibudilast vs placebo, and similar effects were observed for tobacco and cocaine craving. Furthermore, mean subjective ratings of pain were lower in the active ibudilast condition. Our data suggest that ibudilast may be useful for treating opioid use disorders and it may enhance the analgesic effects of oxycodone.
\end{abstract}

Neuropsychopharmacology (2017) 42, I825-1832; doi: I0.1038/npp.2017.70; published online 10 May 2017

\section{INTRODUCTION}

Opioid use disorders (OUDs) involving heroin and prescription opioid analgesics remain a serious global challenge for the health, social, and economic welfare of all affected individuals (Volkow, 2014). Different opioid agonist (methadone, buprenorphine) and antagonist (naltrexone) pharmacotherapies are currently available, but each is associated with certain disadvantages (eg, risk of diversion to illicit use, requirement for detoxification, etc). Therefore, the search for and development of non-opioid treatment medications for OUD is ongoing. A novel approach targets the inflammatory processes involved in opioid addiction, specifically glia cell activation and cytokine release.

\footnotetext{
*Correspondence: Dr VE Metz, Department of Psychiatry, Columbia University Medical Center, New York State Psychiatric Institute, I05 I Riverside Drive, Unit 120, New York, NY 10032, USA, Tel: + I 646774 6139; Fax: + 64677460 I8; E-mail: metzver@nyspi.columbia.edu or metz.verena@gmail.com

Received I4 November 2016; revised I April 2017; accepted 3 April 2017; accepted article preview online 10 April 2017
}

Some studies have suggested that opioids activate glia, which have an essential role in pain states and other processes in rodents, such as opioid reward, dependence, and withdrawal (Watkins et al, 2007; Hutchinson et al, 2007). For example, it has been demonstrated that the release of cytokines following activation of glia results in a suppression of opioid-induced analgesia, which can be prevented or reversed by administration of a glial inhibitor (Watkins $e t$ al, 2005; Hutchinson et al, 2007). Studies in rats have shown that increased withdrawal-induced pain responses are alleviated by medications such as ibudilast that block proinflammatory cytokine production (Johnston et al, 2004; Ledeboer et al, 2007; Lewis et al, 2006). Additionally, glial modulators decreased morphine-induced conditioned place preference in mice (Narita et al, 2006), thus implicating a role of glia in opioid-induced reward and other behavioral effects associated with opioid dependence and abuse liability in animals (for a review, see Cooper et al, 2012). Moreover, Song and Zhao (2001) demonstrated that opioidinduced glial activation reduced chronic opioid analgesia, thereby demonstrating opioid-induced proinflammatory 
glial activation could in turn impact opioid pharmacodynamics. Various opioid actions have been shown to be affected by opioid-induced glial activation, such as the reduction of acute and chronic opioid analgesia, tolerance, hyperalgesia, allodynia, physical dependence, reward, and respiratory depression (Hutchinson et al, 2007, 2008, 2009, 2010). Ibudilast has been efficacious in several rat models of these effects. For example, ibudilast robustly potentiated both acute analgesia to morphine and oxycodone, assessed by a modified Hargreaves method (Hutchinson et al, 2009). Drugs of abuse, such as morphine, elicit increased dopamine levels in the nucleus accumbens, and this is thought to mediate the reward associated with such drugs (Bonci et al, 2003). Rats dependent on morphine showed elevated dopamine levels in the nucleus accumbens following administration of morphine, as determined by microdialysis. Coadministration of ibudilast with morphine to dependent rats reduced the dopamine increase (Bland et al, 2009).

For the first study of glia cell inhibitors in humans with OUD, Cooper et al (2016) examined the effects of ibudilast, which inhibits the proinflammatory cytokine macrophage migration inhibitory factor and certain cyclic nucleotide phosphodiesterases. Ibudilast, also known as AV-411 or MN-166, has been used for more than two decades in Japan and other Asian countries for the treatment of asthma and poststroke dizziness. It is currently being studied for the treatment of chronic neuropathic pain, stimulant and alcohol use disorders, and multiple sclerosis. Moreover, ibudilast has been well tolerated, and has an overall excellent drug safety profile in various patient populations. Cooper et al (2016) stabilized 31 opioid-dependent adults on $120 \mathrm{mg}$ oral morphine for 1 week, and then randomly assigned them to receive either placebo or active ibudilast $(20 \mathrm{mg}$ or $40 \mathrm{mg}$ b.i.d.) for 1 week while being maintained on $120 \mathrm{mg}$ morphine, which was discontinued abruptly in week 3 . Cooper et al (2016) applied a between-subjects design to compare withdrawal symptoms between the placebo and the active ibudilast groups during week 3 . All study participants experienced withdrawal symptoms after abrupt morphine cessation with no significant differences in overall objective or subjective opioid withdrawal symptoms (total Clinical Opiate Withdrawal Scale (COWS) and Subjective Opioid Withdrawal Scale (SOWS) scores, respectively) across the groups. However, when pooling the findings, the placebomedicated group had significantly higher SOWS ratings on the dimensions 'anxious,' 'perspiring,' 'restless,' and 'stomach cramps' than the ibudilast-medicated groups. This effect could not be shown for any other measured parameters. Thus, the effect of ibudilast on opioid withdrawal symptoms was modest. However, the study medication was well tolerated and no serious adverse events occurred in the opioid-dependent volunteers.

Despite these modest results in regard to opioid withdrawal, this first study in humans with opioid dependence suggested that glial cell inhibitors may be able to modulate opioid-induced analgesia and abuse potential (Cooper et al, under revision). Therefore, we initiated the present study that specifically assessed the effects of a higher dose of ibudilast (50 mg b.i.d.) on oxycodone-induced analgesia, selfadministration, subjective responses, and craving. A crossover design was applied to increase the power to detect differences in our measures of interest and volunteers with
OUD were detoxified from opioids before experimental laboratory sessions to more clearly distinguish the effects of ibudilast from placebo.

We hypothesized that (a) oxycodone would produce dosedependent increases in analgesia, positive subjective responses, and breakpoint values in our progressive-ratio drug self-administration procedure, and (b) maintenance on active compared with placebo ibudilast would increase oxycodone-induced analgesia and decrease oxycodone selfadministration and positive subjective effects.

\section{MATERIALS AND METHODS}

Non-treatment-seeking adults with opioid dependence were recruited via advertisements in local newspapers and flyers in public places throughout New York City. After prescreening over the telephone to verify whether they met the basic eligibility criteria, including current opioid dependence (DSM-IV) and no other current alcohol or illicit substance dependence, they were invited for in-person screening visits to the New York State Psychiatric Institute (NYSPI). The volunteers underwent extensive screening procedures including medical and psychiatric examinations, laboratory work, electrocardiogram, and clinical interviews to ensure they had no psychiatric or medical conditions that would interfere with study participation. Urine drug testing (amphetamine, barbiturates, buprenorphine, benzodiazepines, cocaine (benzoylecognine), methamphetamine, methadone, opiates, oxycodone, phencyclidine, marijuana) was also performed multiple times during the screening process. Key exclusion criteria were acute medical or psychiatric disorders and a history of overdose following opioid detoxification.

Eligible volunteers signed informed consent before being admitted to a locked psychiatric inpatient unit for 6 weeks. The study was designed as a randomized placebo-controlled, crossover medication trial, with a 7-day morphine-assisted detoxification, followed by block randomization to either placebo ( $0 \mathrm{mg}$ b.i.d.) or active ibudilast (50 mg b.i.d.) during the first study phase involving laboratory testing sessions. Participants then transitioned to the second medication condition and underwent the same procedures again (see Supplementary Table $1 \mathrm{~A}$ in Supplementary Materials for a representative depiction of the study design).

During the detoxification, participants received sustainedrelease morphine $(60 \mathrm{mg}$ b.i.d.) and a regimen of standing ancillary medications including clonidine, clonazepam, and zolpidem. In addition, supplementary medications (acetaminophen, ibuprofen, milk of magnesia, and additional zolpidem) were available upon request to treat emergent withdrawal symptoms. After this detoxification phase, participants were randomized to receive $50 \mathrm{mg}$ ibudilast or $0 \mathrm{mg}$ ibudilast b.i.d., and stabilized for at least 5-6 days on the full dose of ibudilast before starting laboratory sessions. Under each maintenance dose condition ( $0 \mathrm{mg}$ ibudilast $v s$ $50 \mathrm{mg}$ b.i.d.), the effects of oxycodone (0, 15, and $30 \mathrm{mg} /$ $70 \mathrm{~kg}$, p.o.) were examined during six laboratory sessions that were completed on separate days. For each oxycodone dose, participants completed a 'sample' session on one day followed by a 'choice' session the next day. During the sample session, participants received US\$20 and a dose of oxycodone and subjective, performance, and physiological 
effects were measured both before and after dose administration (Supplementary Table 1B in Supplementary Materials). During the subsequent choice session, participants completed a 10-trial progressive-ratio self-administration task. Subjective, performance, and physiological effects were also completed during the choice session, but these data were not analyzed statistically because different participants selfadministered different amounts of drug. During each of the 10 trials during the self-administration task, participants could choose between 1/10th of the dose of drug they had sampled the previous day or $1 / 10$ th of the money they had sampled. Responses consisted of finger presses on a computer mouse. During the first trial, participants made 25 responses, followed by $50,100,200,400,800,1600,3200,6400$, and 12800 responses each time drug and/or money was selected. The progressive-ratio value increased independently each time drug and/or money were selected. After participants completed the task or stopped responding, they received whatever fraction of the money and dose that they earned.

During all laboratory sessions, participants were monitored and physiological, subjective, and reinforcing effects of oxycodone were measured (see Supplementary Table 1B in Supplementary Materials for overview of time points). Specific dependent variables included physiological parameters (heart rate, systolic blood pressure, diastolic blood pressure, arterial oxygen saturation, pupil diameter), subjective effects (Drug Effects Questionnaire (DEQ), Addiction Research Center Inventory (Hill et al, 1963), SOWS (Handelsman et al, 1987), Visual Analog Mood Scales, Visual Analog Craving Scales (for alcohol, cocaine, heroin, and tobacco)), cognitive performance (Digit Symbol Substitution Test (McLeod et al, 1982), Divided Attention Task (DAT; Miller et al, 1988)), and pain responses from the Cold
Pressor Test (Zacny et al, 1996; Pain Intensity and Bothersomeness VAS Scales, the Short Form-McGill Pain Questionnaire (MPQ; Melzack, 1987), latency to feel pain, and latency to withdraw the hand from the cold water). For a more detailed description of laboratory assessments, please see Comer et al (1999).

In addition, the following safety measures were assessed throughout the study: daily vital signs, weekly or as needed blood chemistries, weekly electrocardiograms, daily urine drug toxicologies, and daily adverse events assessments.

Participants who dropped out or completed the study were provided with treatment referrals and opioid overdose education.

\section{Drugs}

Active ibudilast (10 mg capsules; obtained from MediciNova) was administered in a size 00 opaque capsule with lactose filler prepared by the New York State Psychiatric Institute Research Pharmacy. For placebo ibudilast, identical size 00 capsules containing only lactose filler were administered. A total of five capsules were administered at each dose administration (containing either active ibudilast, for a total of $50 \mathrm{mg}$ per dose administered twice daily or lactose only). These doses were chosen based on the safety and tolerability of ibudilast observed in previous studies using similar dosing regimens for asthma, multiple sclerosis, or other CNS disorders (Feng et al, 2004; Shida et al, 1985; Yagi et al, 2010). Oxycodone $\mathrm{HCl}$ (Oxyfast Immediate-Release Oral Concentrate Solution $(20 \mathrm{mg} / \mathrm{ml})$; Purdue Pharma) was prepared at doses of 0,15 , and $30 \mathrm{mg}$ per $70 \mathrm{~kg}$. The solution was mixed in orange-flavored Gatorade with $1 \mathrm{ml}$ peppermint oil floated on top to mask the taste of the drug. A total
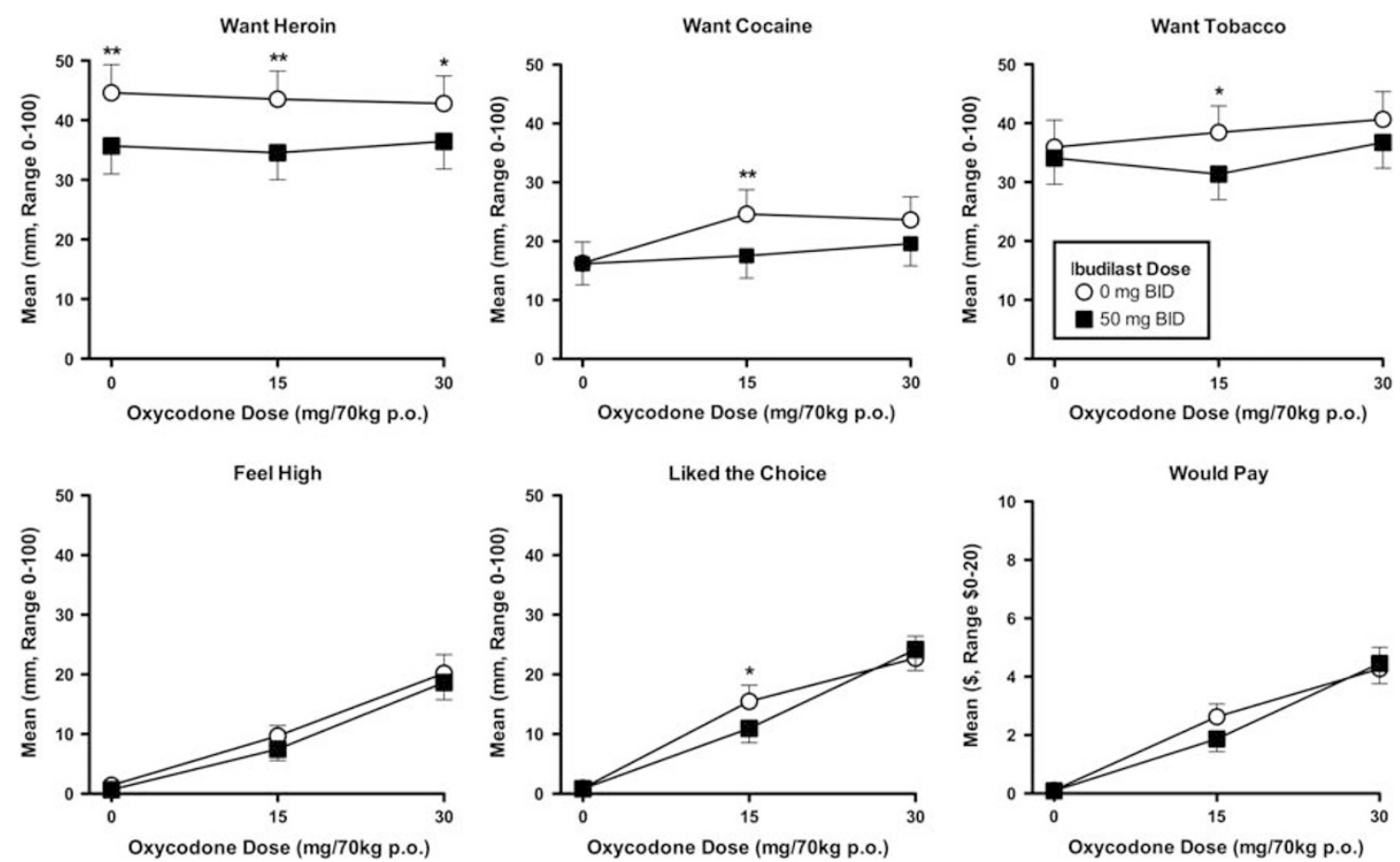

Figure I Select mean subjective ratings on the visual analog scales (VAS) after the administration of 0, I5, and $30 \mathrm{mg} / 70 \mathrm{~kg}$ of oral oxycodone on the sample session days in the active ibudilast (closed squares) vs the placebo ibudilast (open circles) condition. $* p<0.05$ and $* * p<0.0$ l. 
volume of $200 \mathrm{ml}$ was administered at each dosing and consumed within $5 \mathrm{~min}$. We have used a similar procedure in other protocols to successfully mask the flavor of the beverage (Comer et al, 2010).

\section{Statistical Analyses}

Data were analyzed using SPSS for Mac, version 23, PRISM 5 for Mac OS X, and SuperAnova version 1.11. For descriptive parameters, means and standard deviations were calculated. Mixed analyses of variance with planned contrasts were performed to investigate differences in subjective and reinforcing effects of oxycodone under the two ibudilast maintenance conditions ( $0 \mathrm{mg}$ and $50 \mathrm{mg}$ b.i.d.), as well as for physiological, craving, and pain measures. Mean values across the session were evaluated. Peak and trough data were also examined but in the interest of brevity, they are not described here because the overall pattern of effects was similar for these analyses compared with the time-course data.

Block randomization was used to assign participants to active or placebo ibudilast and we tested for potential carryover effects across the maintenance conditions. Specifically, we compared study results from participants who completed the placebo ibudilast maintenance condition first to results from participants who completed the active ibudilast maintenance condition first. For this analysis, we used independent samples $t$-tests and selected measures from each of the key study outcomes (eg, reinforcing effects, subjective effects, and pain ratings). No significant order effects were detected.

For all other analyses, two-tailed testing was conducted, and a $p$-value $<0.05$ was considered statistically significant.

\section{IRB Approval}

The Institutional Review Board of the New York State Psychiatric Institute and the Department of Psychiatry of Columbia University has approved this research study (IRB study number 6021).

\section{RESULTS}

Between October 2012 and November 2015, 75 potential participants were screened. Forty-seven individuals did not meet inclusion criteria $(n=24$ had medical and/or psychiatric diagnoses that were exclusionary, $n=13$ were lost to contact, $n=9$ preferred to participate in another study at the Opioid Laboratory, and one person decided to seek treatment instead of participating in the study), and 28 were enrolled into the study. Of the 28 participants enrolled, 17 dropped out for the following reasons: six participants had behavioral issues on our in-patient unit and were discharged for infractions of rules, seven participants withdrew consent during the detoxification phase of the study, two participants had family emergencies and asked to be discharged, and two participants had cardiovascular issues (labile hypertension and low heart rate, respectively) and were withdrawn from the research by a study physician. These adverse events were considered unlikely to be study medication related, but rather associated with pre-existing conditions. The participants were referred to their primary care physicians, and both medical issues had resolved by the 1 -month follow-up visit.
The 11 study completers were all male: seven were African American, one was Caucasian, two identified as Hispanic/ Latino, and one was multiracial. The sample had a mean age of 45.1 years $(\mathrm{SD}=8.9), 11.7$ years $(\mathrm{SD}=0.9)$ of education, and used opioids for 16.2 years $(\mathrm{SD}=10.0)$ with an average use of 6.4 bags $(S D=2.6)$ of heroin per day before study initiation. All but one participant reported heroin as their drug of choice, whereas one participant primarily used prescription opioids; two participants reported that their primary route of opioid administration was injecting, eight primarily used opioids intranasally, and one primarily used orally. All participants had a lifetime history of smoking cigarettes, and eight participants were regular light cigarette smokers (on average 8 cigarettes per day, range 2-15 cigarettes daily) at the time of study entry. Completers and drop-outs did not differ in any of these basic variables.

\section{Physiological Effects}

No clinically significant changes in any physiological measures occurred when the placebo and active ibudilast doses were compared (see Supplementary Table 2 in Supplementary Materials for detailed results on physiological effects). However, heart rate decreased significantly with increasing oxycodone dose in both the placebo and active ibudilast conditions. Pupil diameter decreased with increasing oxycodone dose $(p<0.001)$, but ibudilast did not have a statistically significant effect on pupil diameter. No significant changes or effects on arterial oxygen saturation, diastolic or systolic blood pressure compared with placebo were observed, regardless of oxycodone or ibudilast condition.

\section{Cognitive Effects}

Oxycodone significantly reduced the mean hit latency $(p<0.01)$ and tracking distance $(p<0.05)$ on the DAT, but ibudilast produced no effects on any of the cognitive effects that were assessed (see Supplementary Table 3 in Supplementary Materials).

\section{Drug Craving}

Heroin craving was consistently high across the oxycodone dose conditions. Although the overall effect of ibudilast on heroin craving was not statistically significant, nor was the ibudilast by oxycodone interaction, planned comparisons revealed significantly less craving for heroin in the active ibudilast condition compared with the placebo condition with all three oxycodone doses $(0 \mathrm{mg}: p<0.001 ; 15 \mathrm{mg}$ : $p<0.001 ; 30 \mathrm{mg}: p=0.016$ ), see Figure 1 .

Craving for cocaine was significantly lower when participants were maintained on active ibudilast compared with placebo in the $15 \mathrm{mg}$ oxycodone condition only $(p=0.002)$; however, no statistically significant result was found for the $30 \mathrm{mg}$ oxycodone condition $(p=0.058)$.

A similar result was observed for nicotine craving in that it was significantly lower under active ibudilast maintenance compared with placebo maintenance in the $15 \mathrm{mg}$ oxycodone condition $(p=0.022)$, but no significant finding was noted in the $0 \mathrm{mg}$ oxycodone $(p=0.521)$ or the $30 \mathrm{mg}$ oxycodone condition $(p=0.114)$. 
Table I Mean (SD) Time Course of Subjective Effects

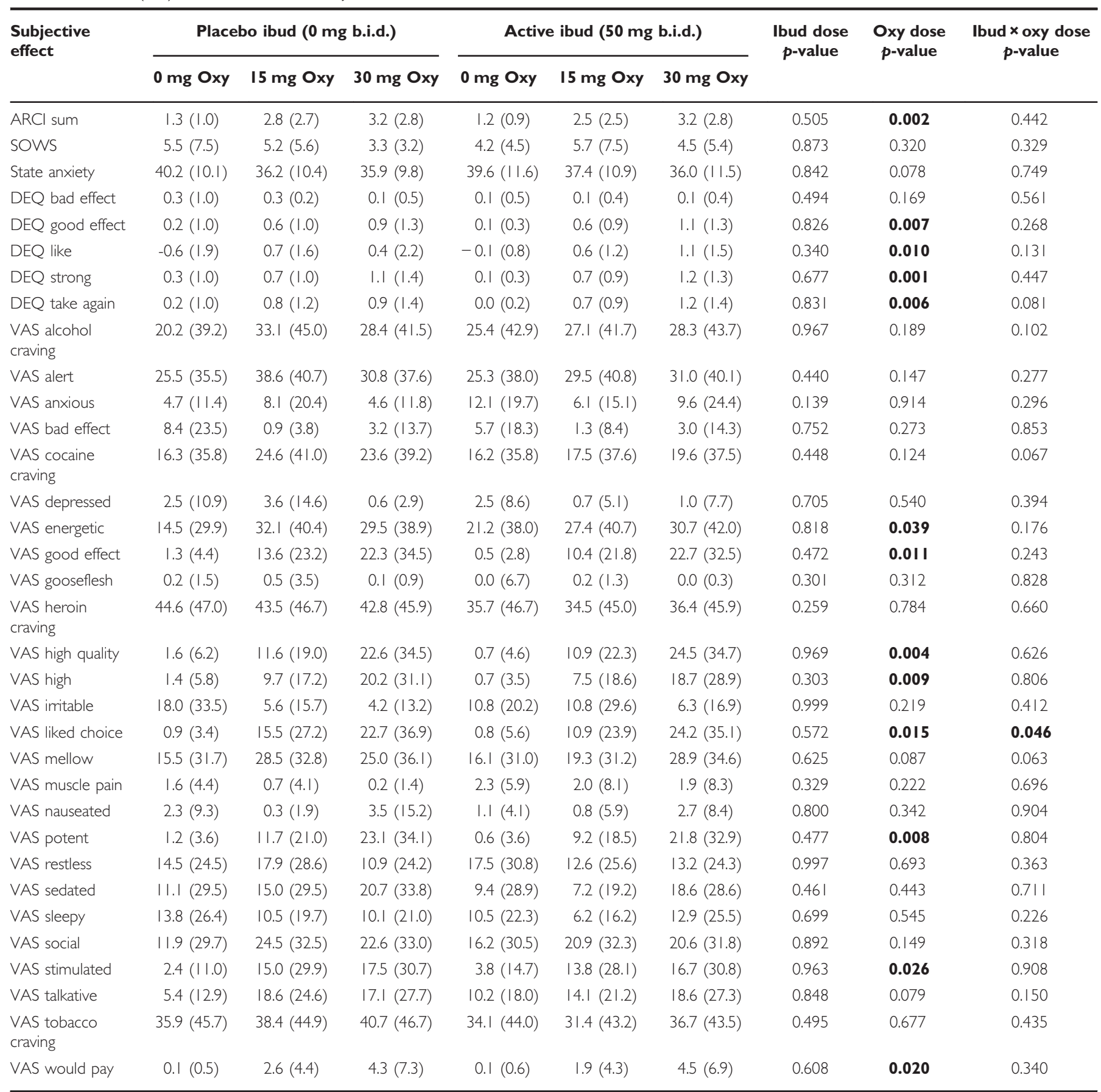

Abbreviations: ARCl, Addiction Research Center Inventory; DEQ, Drug Effects Questionnaire; ibud, ibudilast; oxy, oxycodone; SOWS, Subjective Opioid Withdrawal Scale; VAS, visual analog scales.

Bold values indicate $p$-values $<0.05$.

\section{Subjective Drug Effects}

Oral oxycodone produced significant dose-dependent increases in positive subjective effects on VAS ratings of: 'I feel high' ( $p=0.009)$, 'I liked the choice' $(p=0.015)$, and 'I would pay for the dose' $(p=0.020)$, see Figure 1. On VAS ratings of 'I liked the choice,' the ibudilast $\times$ oxycodone dose interaction was significant $(p=0.046)$, and planned contrasts revealed significant differences between the placebo and active ibudilast conditions in the $15 \mathrm{mg}$ oxycodone condition $(p=0.012)$, but not with either the oxycodone $0 \mathrm{mg}$ $(p=0.956)$ or $30 \mathrm{mg}$ doses $(p=0.384)$. No statistically significant difference between the two ibudilast conditions was found on VAS ratings of 'I would pay'.

Besides the 'liked the choice' item, no significant ibudilast by oxycodone dose interactions were observed. Please see Table 1 for a summary of subjective effects. 


\section{Reinforcing Effects}

Drug breakpoint values differed significantly between active and placebo ibudilast in the $15 \mathrm{mg}$ oxycodone condition $(p=0.035)$, but not in the $30 \mathrm{mg}$ oxycodone condition $(p=0.099)$, see Figure 2 .

\section{Pain Measures}

After the Cold Pressor Test, participants rated their pain on the VAS 'Bothersome' and 'Painful' scales, and with the MPQ; these pain ratings were significantly reduced by the oxycodone dose they had received (main effects of oxycodone dose for 'Bothersome' $p=0.004$, 'Painful' $p=0.008$, and MPQ sum score $p=0.002$ ). However, neither the latency to feel pain nor the latency to withdraw the hand from the cold water were altered in a dose-related manner by oxycodone $(p=0.713, p=0.762)$ or ibudilast $(p=0.699$, $p=0.662)$, and no oxycodone-dose-ibudilast-dose interaction effect was detected $(p=0.578, p=0.552)$.

When participants were maintained on active ibudilast compared with placebo, they reported the pain to be feeling significantly less 'bothersome' in the $30 \mathrm{mg}$ oxycodone condition $(p=0.013)$, but not in the $15 \mathrm{mg}(p=0.289)$ or the $0 \mathrm{mg}(p=0.332)$ conditions, see Figure 3.

On the VAS assessment of 'painful', no differences between placebo and active ibudilast in any of the oxycodone dose conditions reached statistical significance.

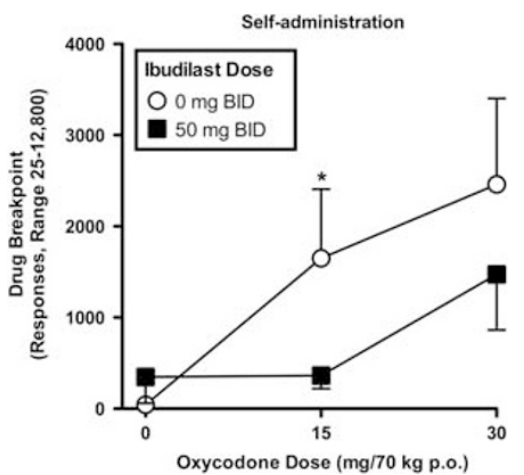

Figure 2 Mean drug breakpoint values for the three available oral oxycodone doses $(0,15$, and $30 \mathrm{mg} / 70 \mathrm{~kg})$ during the choice sessions in the active ibudilast (closed squares) vs the placebo ibudilast (open circles) condition. $* p<0.05$.
The McGill Pain Questionnaire yielded significant differences in pain reductions between the placebo and active ibudilast maintenance conditions in the $15 \mathrm{mg}$ oxycodone condition $(p=0.018)$ and the $30 \mathrm{mg}$ oxycodone condition $(p=0.046)$, but not in the $0 \mathrm{mg}$ oxycodone condition $(p=0.871)$.

\section{DISCUSSION}

Ibudilast was well tolerated, with minimal side effects and no serious adverse events (please see Supplementary Table 4 in Supplementary Materials for details on adverse events). However, it did not robustly alleviate withdrawal symptoms in the current study. One-fourth of the enrolled volunteers left during the detoxification phase of the study. In the first investigation of ibudilast's effects on withdrawal in participants with OUD, only marginal effects on opioid withdrawal symptoms were revealed (Cooper et al, 2016).

In the present study, modest differences were observed in regard to positive subjective effects of oxycodone when participants were maintained on active ibudilast compared with placebo, particularly in the $15 \mathrm{mg}$ oxycodone dose condition. Ibudilast produced a modest attenuation of ratings of drug liking and willingness to pay for the lower oxycodone dose, which are key end points in abuse liability testing. However, this effect was not seen with the higher oxycodone dose; perhaps, a higher ibudilast dose would have been necessary to have an effect on the $30 \mathrm{mg}$ oxycodone dose. This finding leaves us with the possibility that the effects of ibudilast on the subjective responses produced by $15 \mathrm{mg}$ oxycodone were spurious.

The effect of ibudilast on drug breakpoint, as a measure of reinforcement, yielded a more convincing result: when participants were maintained on active ibudilast, responding for drug was significantly reduced in the $15 \mathrm{mg}$ oxycodone condition, but not in the $30 \mathrm{mg}$ oxycodone condition. Heroin craving was also significantly reduced in the active ibudilast condition compared with placebo, independent of the oxycodone dose ingested. Cocaine and tobacco craving were reduced in the active oxycodone conditions, but not in the $0 \mathrm{mg}$ oxycodone condition. The effects on craving for these drugs might be diminished because neither cocaine nor tobacco was the participants' primary drug of choice. In addition, their most recent use of cocaine before study entry
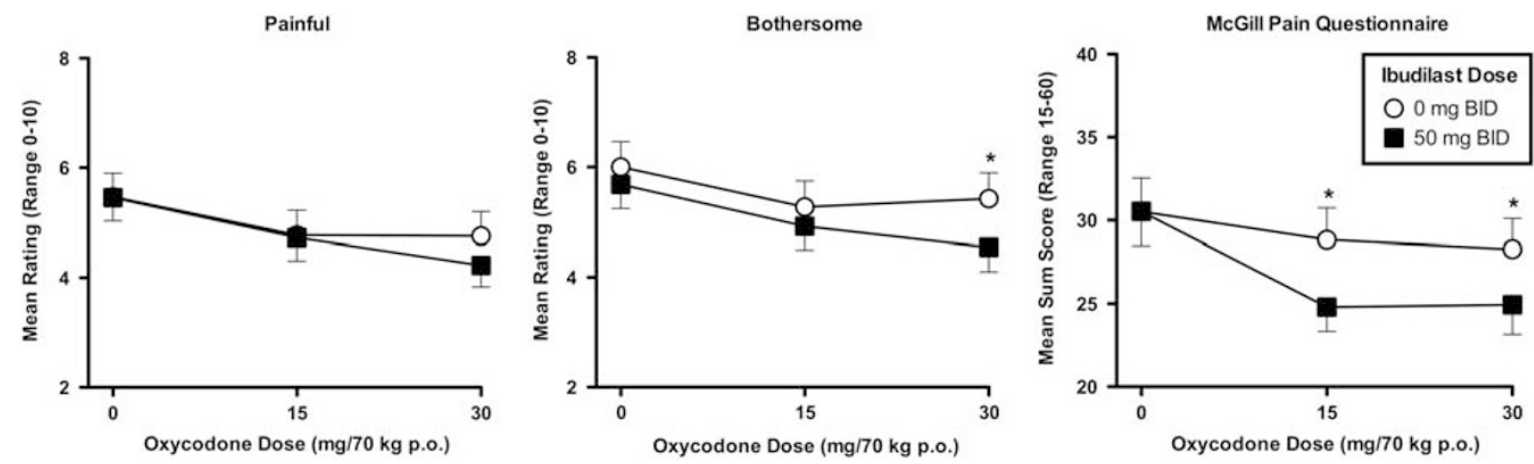

Figure 3 Mean pain ratings on the 'Painful' and 'Bothersome' visual analog scales (VAS) as well as on the McGill Pain Questionnaire after administration of 0, 15 , and $30 \mathrm{mg} / 70 \mathrm{~kg}$ oral oxycodone on the sample session days in the active ibudilast (closed squares) vs the placebo ibudilast (open circles) condition. $* p<0.05$. 
was not consistent across the study participants. Although no participant had a current cocaine use disorder at the time of screening, all participants had lifetime exposure to nicotine and all but one indicated prior cocaine use. Moreover, current smokers received nicotine replacement and/or could use e-cigarettes throughout the study. Alcohol craving seemed to be unaffected by oxycodone and ibudilast; no changes from placebo were observed in any of the medication conditions (see Table 1). However, alcohol abuse and dependence were exclusionary in this study.

Interestingly, active ibudilast seemed to enhance oxycodone-induced analgesic effects compared with placebo; however, the significance of the findings was not consistent across the three analgesia scales. In addition, the objective measures of analgesia (latency to feel pain and latency to withdraw the hand from the cold water) were not significantly altered by either oxycodone or ibudilast. Inspection of the data revealed a great deal of individual variability both across participants and laboratory sessions for these end points. However, our results overall seem to be consistent with those found in rodent models, where ibudilast had an additive antinociceptive effect when administered with morphine in the tail-flick test (Lilius et al, 2009), and even reversed allodynia in rats with central nervous system damage (Ellis et al, 2014). At this point, we also need to consider the lack of women in our sample. In general, women show a larger analgesic response to opioids compared with men; to achieve equivalent pain relief, men require a $30-40 \%$ greater dosage of morphine (Pleym et al, 2003). Thus, we speculate that women would require a lower dose of ibudilast than men to show the enhanced opioid analgesic effects. Further studies are needed to shed light on the dose of ibudilast that might be most effective in women.

Although it would be tempting to speculate that ibudilast was producing its effects on opioid-mediated responses through inhibition of glial activation because the results of our study were somewhat consistent with the preclinical literature on this topic, we are not able to do so at this time because we do not have a reliable objective method of detecting glial activity in the brain. Nevertheless, our findings suggest that ibudilast may be useful for altering opioidinduced positive subjective responses, opioid self-administration, drug craving, and pain in adults with OUDs. Thus, the data support the potential clinical use of ibudilast as a treatment option for individuals with OUD. In particular, ibudilast may be useful as a medication given during and after opioid detoxification because it reduces craving and therefore could be helpful for maintaining abstinence. Moreover, further studies investigating ibudilast in combination with opioids for treating pain is warranted. Because ibudilast increased the analgesic effects of oxycodone, lower doses of the opioid agonist potentially could be used when given in combination with ibudilast. At the same time, ibudilast may reduce the abuse liability of an opioid agonist because it reduces craving and drug liking.

\section{FUNDING AND DISCLOSURE}

This study was supported by National Institute on Drug Abuse grants DA09236 and DA037842. Supplementary funding and ibudilast were provided by Medicinova. The study sponsors had no role in data analysis and interpretation, or the writing of the manuscript. The authors declare no conflict of interest.

\section{ACKNOWLEDGMENTS}

We thank the team members who put a great deal of effort into working on the study, especially Rachel Luba, Claudia Tindall, Janet Murray, the clinical staff at our in-patient unit 5-South, and the NYSPI pharmacy. We further thank the study participants for taking part in the research. Finally, we also thank their Division chiefs, Drs Herbert Kleber and Francis Levin, who supported the implementation of this project.

\section{REFERENCES}

Bland ST, Hutchinson MR, Maier SF, Watkins LR, Johnson KW (2009). The glial activation inhibitor AV411 reduces morphineinduced nucleus accumbens dopamine release. Brain Behav Immun 23: 492-497, PMCID: PMC2783267.

Bonci A, Bernardi G, Grillner P, Mercuri NB (2003). The dopamine-containing neuron: maestro or simple musician in the orchestra of addiction? Trends Pharmacol Sci 24: 172-177.

Comer SD, Collins ED, MacArthur RB, Fischman MW (1999). Comparison of intravenous and intranasal heroin selfadministration by morphine-maintained humans. Psychopharmacology (Berl) 143: 327-338.

Comer SD, Sullivan MA, Vosburg SK, Kowalczyk WJ, Houser J (2010). Abuse liability of oxycodone as a function of pain and drug use history. Drug Alcohol Depend 109: 130-138.

Cooper ZD, Johnson KW, Pavlicova M, Glass A, Vosburg SK, Sullivan MA et al (2016). The effects of ibudilast, a glial activation inhibitor, on opioid withdrawal symptoms in opioid-dependent volunteers. Addict Biol 21: 895-903.

Cooper ZD, Jones JD, Comer SD (2012). Glial modulators: a novel pharmacological approach to altering the behavioral effects of abused substances. Expert Opin Investig Drugs 21: 169-178.

Ellis A, Wieseler J, Favret J, Johnson KW, Rice KC, Maier SF et al (2014). Systemic administration of propentofylline, ibudilast, and (+)-naltrexone each reverses mechanical allodynia in a novel rat model of central neuropathic pain. J Pain 15: 407-421.

Feng J, Misu T, Fujihara K, Sakoda S, Nakatsuji Y, Fukaura H et al (2004). Ibudilast, a nonselective phosphodiesterase inhibitor, regulates Th1/Th2 balance and NKT cell subset in multiple sclerosis. Mult Scler 10: 494-498.

Handelsman L, Cochrane KJ, Aronson MJ, Ness R, Rubinstein KJ, Kanof PD (1987). Two new rating scales for opiate withdrawal. Am J Drug Alcohol Abuse 13: 293-308.

Hill HE, Haertzen CA, Wolbach AB Jr, Miner EJ (1963). The addiction research center inventory: Appendix. I. Items comprising empirical scales for seven drugs. II. Items which do not differentiate placebo from any drug condition. Psychopharmacologia 4: 184-205.

Hutchinson MR, Bland ST, Johnson KW, Rice KC, Maier SF, Watkins LR (2007). Opioid-induced glial activation: mechanisms of activation and implications for opioid analgesia, dependence, and reward. Scientific World J 7: 98-111.

Hutchinson MR, Coats BD, Lewis SS, Zhang Y, Sprunger DB, Rezvani N et al (2008). Proinflammatory cytokines oppose opioid-induced acute and chronic analgesia. Brain Behav Immun. 22: 1178-1189.

Hutchinson MR, Lewis SS, Coats BD, Skyba DA, Crysdale NY, Berkelhammer DL et al (2009). Reduction of opioid withdrawal and potentiation of acute opioid analgesia by systemic AV411 (ibudilast). Brain Behav Immun 23: 240-250. 
Hutchinson MR, Zhang Y, Shridhar M, Evans JH, Buchanan MM, Zhao TX et al (2010). Evidence that opioids may have toll-like receptor 4 and MD-2 effects. Brain Behav Immun 24: 83-95.

Johnston IN, Milligan ED, Wieseler-Frank J, Frank MG, Zapata V, Campisi J et al (2004). A role for proinflammatory cytokines and fractalkine in analgesia, tolerance, and subsequent pain facilitation induced by chronic intrathecal morphine. J Neurosci 24: $7353-7365$

Ledeboer A, Hutchinson MR, Watkins LR, Johnson KW (2007). Ibudilast (AV-411). A new class therapeutic candidate for neuropathic pain and opioid withdrawal syndromes. Expert Opin Investig Drugs 16: 935-950.

Lewis SS, Hutchinson MR, Coats BD, Brzeski AL, Maier SF, Watkins LR et al (2006). AV411, a blood-brain barrier permeable glial activation inhibitor, reduces morphine withdrawal behaviors in rats. Proc Soc Neurosci 765: 762.

Lilius TO, Rauhala PV, Kambur O, Kalso EA (2009). Modulation of morphine-induced antinociception in acute and chronic opioid treatment by ibudilast. Anesthesiology 111: 1356-1364.

McLeod DR, Griffiths RR, Bigelow GE, Yingling J (1982). An automated version of the Digit Symbol Substitution Test (DSST). Behav Res Meth Instr 14: 463-466.

Melzack R (1987). The short-form McGill Pain Questionnaire. Pain 30: 191-197.

Narita M, Miyatake M, Narita M, Shibasaki M, Shindo K, Nakamura A et al (2006). Direct evidence of astrocytic modulation in the development of rewarding effects induced by drugs of abuse. Neuropsychopharmacology 31: 2476-2488.

Miller TP, Taylor JL, Tinklenberg JR (1988). A comparison of assessment techniques measuring the effects of methylphenidate, secobarbital, diazepam and diphenhydramine in abstinent alcoholics. Neuropsychobiology 19: 90-96.

Pleym H, Spigset O, Kharasch ED, Dale O (2003). Gender differences in drug effects: implications for anesthesiologists. Acta Anaesthesiol Scand 47: 241-259.

Shida T, Kawakami Y, Mue S (1985). Clinical studies of KC-404 for treatment of adult bronchial asthma. Evaluation by multiinstitution open trial. Kiso to Rinsho 19: 65-81.

Song P, Zhao ZQ (2001). The involvement of glial cells in the development of morphine tolerance. Neurosci Res 39: 281-286.

Volkow ND (2014). Prescription opioid and heroin abuse. Available at https:/www.drugabuse.gov/about-nida/legislative-activities/ testimony-to-congress/2015/prescription-opioid-heroin-abuse (Accessed 16 September 2016).

Watkins LR, Hutchinson MR, Johnston IN, Maier SF (2005). Glia: novel counter-regulators of opioid analgesia. Trends Neurosci 28: 661-669.

Watkins LR, Hutchinson MR, Milligan ED, Maier SF (2007). 'Listening' and 'talking' to neurons: implications of immune activation for pain control and increasing the efficacy of opioids. Brain Res Rev 56: 148-169.

Yagi K, Tada Y, Kitazato KT, Tamura T, Satomi J, Nagahiro S (2010). Ibudilast inhibits cerebral aneurysms by down-regulating inflammation-related molecules in the vascular wall of rats. Neurosurgery 66: 551-559, discussion 559.

Zacny JP, McKay MA, Toledano AY, Marks S, Young CJ, Klock PA et al (1996). The effects of a cold-water immersion stressor on the reinforcing and subjective effects of fentanyl in healthy volunteers. Drug Alcohol Depend 42: 133-142.

Supplementary Information accompanies the paper on the Neuropsychopharmacology website (http://www.nature.com/npp) 